\title{
IoT Framework Based Health Care System for Elderly and Disabled People
}

\author{
Syed Misbahuddin*, Humam Orabi, Ryan Fatta, Mishary Al-Juhany and Azzam Almatrafi \\ Electrical Engineering Department \\ College of Engineering and Islamic Architecture \\ Umm Al-Qura University, Makkah, Saudi Arabia \\ *doctoyedmisbah@yahoo.com
}

\begin{abstract}
Approximately $15 \%$ of world population faces some sort of disability which effects their social life and make them overly dependent on the others. Similarly old aged peoples' cognitive, physical and psychological health is negatively affected due to aging. To increase the disabled and old aged peoples' functional capabilities, several IT-enabled devices have been designed. Internet of Things (IoT) is a new paradigm in which "things" are connected to Internet for control applications. In this paper an IoT based health care system has been developed to help disabled or old aged people. In this system elderly or disabled people can communicate their urgent needs to the health care staff in the form of specific important prerecorded audio messages. These messages can be played by touching their smart phone scree on IoT interface. Also, with the suggested design, the elderly people can control the home appliances from one location using their smart phones.
\end{abstract}

Keywords-Internet of Things, Embedded PCs, IoT solutions for disabled people

\section{INTRODUCTION}

Around $15 \%$ of world population faces some sort of disability as reported by world health organization [1]. Handicapped or disabled people require 24 hours attention. This amount of attention needs lots of human efforts. Advances in the digital era have resulted in many innovations in technology that have help improve the quality of life for the disabled and old aged community. The Internet of Thing (IoT) is a new paradigm aimed to provide very promising directions in the field of IT. Internet of Things aims to connect virtually "everything" to Internet. Luigi et al presented a thorough review of current trends in IoT [2]. The Internet of Things can play a vital role to provide solutions to disabled and old aged people. With IoT enabled solutions, the disabled or senior citizens can experience quality life and play their role in the society.

In the original paradigm of IoT, connectivity to Internet is implemented by sensor devices such as RFID (Radio Frequency Identification). In other words, IoT's main supporting technology is a wireless sensor network and radio frequency identification technology. However, newly surfaced embedded PCs such as Raspberry Pi, Galileu or similar Embedded computing units can also be used in IoT infrastructure [4-6]. The Internet of Things enables new means of communication between people, things and environment. Therefore, IoT based solutions may help old aged people and handicapped people to handle their tasks in much easier way [3]. In this paper, we are presenting an IoT based solution that will help elderly and handicapped to communicate their needs to remotely located health care people in the form of short messages. These messages can be played by touching corresponding buttons on the smart phones. Similar solution has been presented in [4], where a voice chip controlled by a Complex Programmable Logic Device (CPLD) has been used. In this approach the message duration is very limited. Our contribution will help the disabled or old aged people to communicate their needs in form of short prerecorded standard messages over the Internet. Rest of the paper is organized as follows: section II reviews the Raspberry Pi as embedded PC, section III describes the software and hardware architecture of the proposed model. Finally, the paper is concluded in section IV.

\section{RASPBERRY PI AS EMBEDDED PC}

Raspberry Pi (Rpi) has emerged in the context of IoT. Rpi is a handheld very low cost single board computer. It runs a Linux version of operating system. Rpi can provide complete computing solution as available on any standard desktop Linux machine. It's onboard General Purpose Input-output (GPIO) connectors allows Rpi to interface with external circuitry. Rpi's Model B has 512 Mb RAM, 2 USB ports, an Ethernet port and 26 extension connector for interfacing with external hardware. Rpi has a Broadcom BCM2835 system-on-chip (SoC) which includes an ARMl176JZF-S $700 \mathrm{MHz}$ processor, Video Core IV GPU and some memory. Rpi card contains special interface slots called Serial Camera Interface (CSI) and Display Interface (DSI) for connecting special camera and display respectively. Rpi expansion header consists of two rows of pins. Most of these pins are general purpose input/output pins (GPIOs), which can be used to connect Rpi to other electronic devices or external hardware. Recently Raspberry Pi 2 Model B has emerged as second generation Raspberry Pi. Compared to the Raspberry Pi 1 it has a 900MHz quad-core ARM Cor-tex-A7 CPU and 1 GB RAM. On Raspberry Pi 2, total number of GPIO pins have been increased to 40. Raspberry Pi uses Secure Digital (SD) nonvolatile memory card to hold the operating system and data. There are several freely downloadable Linux versions available at Raspberry Pi foundation's website [7]. All these Linux versions carry features of standard Linux 


\section{A. General Purpose IO (GPIO)}

A GPIO line on Rpi can be derived by sending high or low logic pulses by using Python commands for example, GPIO.output(7,True) or PIO.output(7,False) can to send True or False Logic to GPIO pin 7 respectively. The GPIO can also be controlled by using WiringPi C library especially written for Rpi [11]. A simple LED connection to GPIO number 1 is shown in Figure 1.

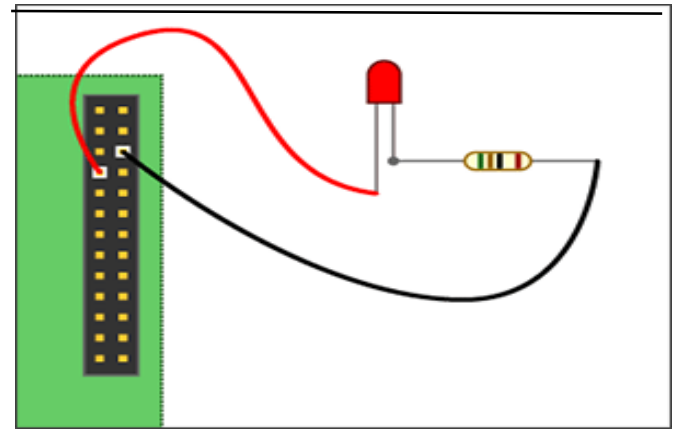

Figure 1: Simple LED connection to GPIO 7

\section{B. WebIOPi}

WebIOPi is a Representational State Transfer (REST) framework which extends the Rpi's GPIO over the Internet or Intranet to a distanced user via a browser [8]. WebIOPi attributes the IoT feature in Raspberry Pi platform. The client can use a PC, smart phone or tablet to access the GPIO interface of Rpi. The GPIO interface displayed on the client device is shown in Figure 2.

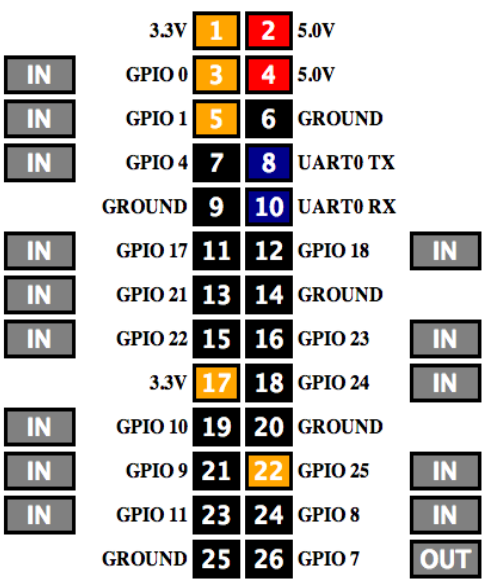

Figure 2: Rpi’s GPIO Interface via WebIOPi

The user can choose input or output mode of any programmable GPIO pin through the web browser running on any device by simply clicking the button next to the GPIO shown in Figure 2. For output GPIO, logic value can be set or reset by clicking on the button for the GPIO pins on the smart phone on the client's PC.

\section{Smart Phone App for GPIO}

Recently an app called "Rpi Automation" has been developed for Android smart phone. The application is available for free download over google play store. Rpi Automation app extends GPIO interface over the smart phone and allows sending high or low logic levels to remote Rpi by touching the buttons on the smart phone. For using Rpi Automation, smart phone and Rpi should be connected via wifi router and must have same IP range. Figure 3 shows Rpi automation app interface on the smart phone.

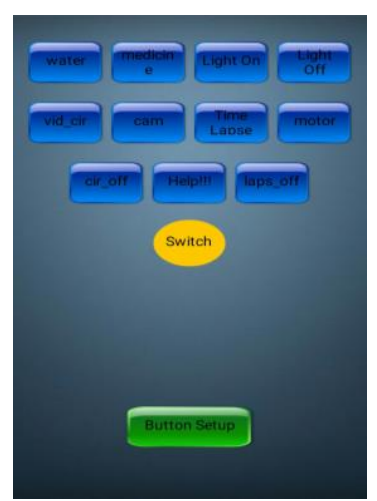

Figure 3: Rpi Automation interface on Mobile Phone

\section{VOICE MESSAGE CONTROL ON RPI}

Elderly or disabled people need frequent attention. The nurses or health care staff may not be around them all the times. The elderly or disabled people may not speak loudly or make phone calls to draw the attention of the care taking staff. We are presenting a Raspberry Pi based solution to help elderly and disabled people in which they can communicate their needs in the form of small pre-recorded standard messages. These messages can be played by touching buttons on their smart phone. These massages can be heard on the remote Raspberry Pi being carried by the remote health care staff. Various buttons can be programmed corresponding to different types of audio messages.

\section{A. Methodology}

To attribute the voice command control on the Rpi we used Espeak open source speech synthesizer software [10]. Espeak provides text-to-phoneme translation. In our design we have used GPIO pins to play customized messages. In order to control messaging system, an elderly or disabled person will access Rpi's GPIO by running Rpi Automation app on his or her smart phone. The interface will use the WebIoPi framework installed at Rpi which extends Rpi's GPIO interface to the smart phones. A particular message can be selected through a GPIO pin. A message $x$ will be played on the Rpi if GIPO $\mathrm{x}$ is touched on the smart phone. The messages can be heard by the remote health care staff on the audio interface available of Rpi. The developed prototype system model is shown in Figure 4. 

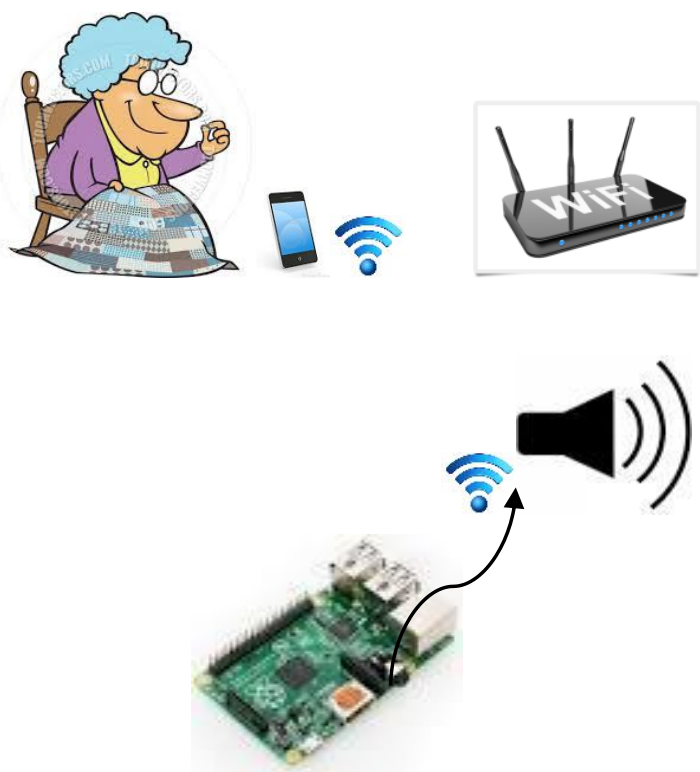

Figure 4: The suggested system for voice message communication

Figure 4 shows that an old lady is using her smart phone to play a specific message on the remote Raspberry Pi. The message is played on the speaker connected to the Rpi's audio jack. We used a Wi-Fi router which assigns same IP range to smart phone and to Rpi. A Wi-Fi dongle is needed at Rpi because it does not contain onboard Wi-fi device.

\section{B. Experimental Results}

For the proof of concepts, we used three GPIO pins 17, 22 and 27 on Raspberry $\mathrm{Pi}$ to implement three representative messages shown in Table 1. Rpi Automation app running on the smart phone presents the buttons corresponding to the GPIO pins. A messages is played on the Rpi whenever a corresponding buttons on the smart phone is touched.

Table 1: GPIO pin to Message Relationship

\begin{tabular}{|l|l|}
\hline GPIO Number & Message \\
\hline 17 & $\begin{array}{l}\text { "Hello, bring me water } \\
\text { please" }\end{array}$ \\
\hline 22 & $\begin{array}{l}\text { "I want my medicine } \\
\text { please" }\end{array}$ \\
\hline 27 & "Help Help Help!!" \\
\hline
\end{tabular}

Figure 5 shows the flowchart for implementing the developed algorithm.

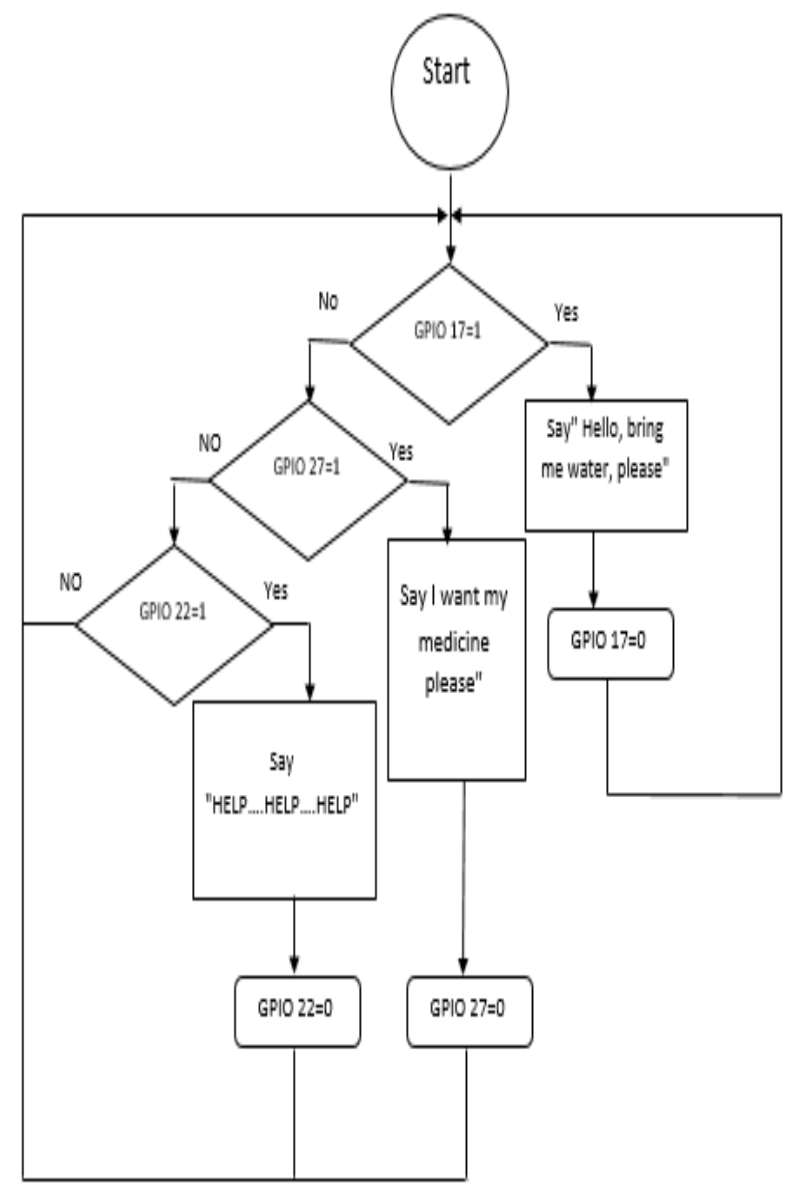

Figure 5: Voice activation algorithm

The messages can be recorded in any language because Espeak utility converts Roman text to voice. The algorithm implemented in Python language which begins by sensing the logic status on the related GPIOs. For example logic status on GPIO 17 will change to logic High if an elderly person touches the button on smart phone corresponding to GPIO 17. For example, the message "Hello, bring me water please" will be played on the Rpi carried by the health care personnel. This message can be heard on the headphone or speaker attached to the audio jack on Raspberry Pi. The health care staff can carry the Rpi unit in his pocket and can hear the message on his headphone as long he is within the Wi-Fi range with the smart phone. The system is scalable in coherent manner for accommodating more required audio messages.

\section{Controlling Home Appliances via Smart Phone}

A GPIO pin on Rpi can be used to energize relays. We have used a 5V 4-Channel Relay interface board to control the appliances such as light bulbs, air conditioner etc. Appliance $\mathrm{x}$ can be associated with GPIO $\mathrm{x}$. Whenever an elderly person touches a specific GPIO to set logic 1 or 0 , the corresponding appliance will be turned on or off respectively. 


\section{CONCLUSION}

This paper has suggested a Raspberry Pi platform based system for helping elderly and disabled person who cannot speak loudly or cannot move to control the appliances. The proposed system is based on the IoT framework running on the Raspberry Pi. The presented schemed helps communicate the important messages to the health care staff in an elderly care unit where patients cannot speak loudly or easily. They can inform about their needs by simply touching some buttons on their smart phones. Also, they can control the appliances by touching the but-tons. If a public IP address is assigned to Raspberry $\mathrm{Pi}$, then elderly people can play the prerecorded audio message over the Internet. The system's future expansion can allow communicating the message anywhere in the world. The Rpi on which the messages will be played can be with the family doctors or with health care people in remote health care units or in any emergency unit.

\section{ACKNOWLEDGMENT}

Authors would like to acknowledge Umm Al-Qura University, Makkah Saudi Arabia for providing the research environment for conducting this experiment.

\section{REFERENCES}

[1] World Health Organization (WHO). World report on disability; June 2011

[2] Luigi Atzori Antonio Iera and Giacomo Morabito, "The Internet of Things: A survey," in Computer Networks, Vol. 54, 2010.

[3] Mari Carmen Domingo, "An overview of the Internet of Things for people with disabilities", Volume 35, Issue 2, March 2012, Pages 584596

[4] www.uwyo.edu/electrical/faculty-staff/steven.../uw_proj_thomas.pdf

[5] Internet of Things: New Promises for Persons with Disabilities, G3ict publications and reports, July 2015

[6] http://arduino.cc/en/ArduinoCertified/IntelGalileo

[7] http://arduino.cc/en/ArduinoCertified/IntelGalileo

[8] https://code.google.com/p/webiopi/

[9] http://play.google.com

[10] http://espeak.sourceforge.net/

[11] http://wiringpi.com 